\title{
Embolismo tumoral pulmonar microvascular en paciente con tumor urotelial
}

\author{
Microvascular pulmonary tumor embolism \\ in a patient with urothelial tumor
}

\author{
B. de Escalante Yangüela ${ }^{1}$, J. Rubio Gracia ${ }^{1}$, G. Muñoz González ${ }^{2}$, B. Gracia Tello${ }^{1}$, \\ M. Rodero Roldán ${ }^{1}$, V. Alastrué del Castaño ${ }^{1}$
}

\section{RESUMEN}

Los embolismos tumorales pulmonares (ETP) representan una causa poco frecuente de disnea en pacientes oncológicos. La mayoría se diagnostican en pacientes con tumores avanzados, sobre todo localizados en mama, pulmón o estómago. Existen pocos casos publicados en pacientes con tumores uroteliales.

Presentamos el caso de un varón de 69 años, sin diagnóstico previo de cáncer, que ingresó por disnea subaguda, con sospecha clínica inicial de tromboembolismo pulmonar (TEP). El paciente falleció al $5^{\circ}$ día del ingreso. En la autopsia se comprobó la existencia de un tumor en pelvis renal izquierda con metástasis hepáticas y linfoganglionares múltiples y una extensa embolia pulmonar microvascular que afectaba a gran parte de los capilares y vasos sanguíneos de mediano calibre de ambos pulmones. Los ETP se consideraron responsables de la insuficiencia respiratoria progresiva y se interpretaron como la causa última de la muerte.

La presentación clínica más frecuente de los ETP es la disnea, a menudo se confunden con el TEP y el diagnóstico no resulta fácil. Su pronóstico es muy malo, con una elevadísima mortalidad y su confirmación habitualmente es post-mortem.

Palabras clave. Embolismo tumoral pulmonar. Carcinoma urotelial micropapilar. Autopsia.

\begin{abstract}
Pulmonary tumor embolisms (PTE) are an infrequent cause of dyspnea in oncological patients. The majority are diagnosed in patients with advanced tumors, above all localized in the breast, lung or stomach. There are few published cases involving patients with urothelial tumors.

We present the case of a 69 year-old male, without a previous diagnosis of cancer, who was admitted due to subacute dyspnea, with clinical suspicion of pulmonary thromboembolism (PT). The patient died on the fifth day of admission. The autopsy confirmed the existence of a tumor in the left renal pelvis with hepatic and lymphoganglionary metastasis and an extensive microvascular pulmonary embolism that affected a large part of the capillaries and medium-caliber blood vessels of both lungs. PTE were considered responsible for the progressive respiratory failure and as the final cause of death.
\end{abstract}

The most frequent clinical presentation of PTE is dyspnea. They are often mistaken for PT and diagnosis is not easy. Their prognosis is very bad, with extremely high mortality and confirmation is usually post-mortem.

Keywords. Pulmonary tumor embolism. Micropapillary urothelial carcinoma. Autopsy.
1. Servicio de Medicina Interna. Hospital Clínico Universitario Lozano Blesa. Zaragoza.

2. Servicio de Anatomía Patológica. Hospital Clínico Universitario Lozano Blesa. Zaragoza.

Recepción: 1 de febrero de 2015

Aceptación provisional: 2 de marzo de 2015

Aceptación definitiva: 10 de marzo de 2015
Correspondencia:

Begoña de Escalante Yangüela

Servicio de Medicina Interna

Hospital Clínico Universitario Lozano Blesa 50009 Zaragoza

Email: bescalantester@gmail.com 


\section{INTRODUCCIÓN}

El embolismo tumoral pulmonar es un cuadro poco frecuente y de difícil diagnóstico en vida del paciente. La mayoría de los casos se presentan en pacientes con tumores avanzados de distintas localizaciones y raramente en tumores uroteliales ${ }^{1}$. Se confunde a menudo con el tromboembolismo pulmonar ${ }^{2}$ y su diagnóstico suele ser tardío, la mayor parte tras estudios posmortem. Presentamos un paciente con émbolos tumorales múltiples asociados a un tumor urotelial no diagnosticado con anterioridad.

\section{CASO CLÍNICO}

Varón de 69 años, exfumador desde 3 años antes (50 paquetes/año). Ingresó por disnea de un mes de evolución, progresiva hasta hacerse de mínimos esfuerzos y, en ocasiones, de reposo, sin ortopnea. No refería tos, expectoración ni fiebre, pero sí pérdida de $5 \mathrm{~kg}$ de peso. Estaba siendo estudiado en el servicio de Urología por hematuria ocasional. Al examen físico se comprobó saturación de oxígeno del $88 \%$, ligera palidez y taquipnea leve. La auscultación cardiopulmonar mostró tonos cardíacos rítmicos, sin soplos, a 107 latidos por minuto y crepitantes teleinspiratorios en bases. Se palparon adenopatías supraclaviculares izquierdas y laterocervicales de $1-2 \mathrm{~cm}$ y hepatomegalia de $4 \mathrm{~cm}$. Las extremidades inferiores no mostraron edemas ni signos de trombosis venosa profunda.

En las pruebas realizadas en el servicio de Urgencias destacaron D-Dímero de $26778 \mu \mathrm{g} / \mathrm{l}$ $(\mathrm{N}<500)$, alcalosis respiratoria con saturación de O2: $92 \%$ y electrocardiograma con taquicardia sinusal a 96 latidos por minuto, con patrón S1Q3T3. La radiografía de tórax fue normal.

Con la sospecha de tromboembolismo pulmonar (TEP) se inició tratamiento con oxigenoterapia y enoxaparina a dosis terapéuticas. Se realizó gammagrafía pulmonar de ventilación/ perfusión (GV/Q) por referir alergia a contrastes yodados, que resultó de baja probabilidad de TEP. El eco-doppler de extremidades inferiores descartó trombosis venosa profunda.

Se revisaron los estudios realizados en el servicio de Urología antes de su ingreso y la ecografía de vías urinarias evidenció quistes renales y próstata hipertrófica. En la TC abdominal sin contraste, se apreciaron lesiones hepáticas compatibles con metástasis, escasa ascitis, adenopatías retroperitoneales y lesión en pelvis renal izquierda sospechosa de neoplasia urotelial.
En los análisis de sangre destacaron urea: $0,65 \mathrm{~g} / \mathrm{l}$; creatinina: $1,45 \mathrm{mg} / \mathrm{dl}$; LDH $1059 \mathrm{U} / \mathrm{L}$; alfa-2 globulinas 19,3\%; hemograma normal; VSG $107 \mathrm{~mm}$ a la $1^{\mathrm{a}}$ hora; fibrinógeno $841 \mathrm{mg} / \mathrm{dl} \mathrm{y} \mathrm{va-}$ rios marcadores tumorales elevados de forma inespecífica, siendo el PSA normal. Orina: hematíes > 100/campo.

Pendiente de la realización de pruebas complementarias, incluidos nueva ecografía de vías urinarias, ecocardiograma transtorácico y biopsia de adenopatías supraclaviculares, entre otras, el paciente presentó fiebre; se realizaron hemocultivos y urocultivo que fueron negativos; nueva radiografía de tórax (sin cambios) y se inició tratamiento antibiótico empírico. Se produjo un incremento brusco de la disnea con taquipnea a $44 \mathrm{rpm}$ y aparición de ingurgitación yugular importante, precisando oxígeno a flujos altos. Se planteó traslado a UCI y pocos minutos después sufrió parada cardiorrespiratoria con maniobras de reanimación cardiopulmonar inefectivas, falleciendo al $5^{\circ}$ día del ingreso.

La autopsia reveló una tumoración de $3 \mathrm{~cm}$ en pelvis renal, asociada a múltiples lesiones ocupantes de espacio en hígado y conglomerados adenopáticos de aspecto metastásico en región supraclavicular, periaórtica, mediastínica y peripancreática. Ambos pulmones mostraron aspecto congestivo, pero sin lesiones tumorales macroscópicas ni signos de tromboembolismo de la arteria pulmonar.

Microscópicamente se constató que la neoplasia primitiva del paciente se localizaba en pelvis renal y mostraba características histológicas de carcinoma urotelial micropapilar (Fig.1). Se observó importante invasión linfovascular y perineural. Todos los conglomerados adenopáticos y las lesiones hepáticas eran metastásis de la neoplasia urotelial. El hallazgo más relevante fue la presencia de una extensa embolia pulmonar microvascular que afectaba a gran parte de los capilares y vasos sanguíneos de mediano calibre de ambos pulmones, ocupando en muchos casos más del $80 \%$ de la luz y, frecuentemente, el 100\% (Figs. 2a y 2b); esta oclusión del lecho capilar pulmonar se asociaba con edema y congestión vascular, se consideró responsable de la insuficiencia respiratoria progresiva y se interpretó como la causa última de la muerte.

La citología de orina, recibida postmortem, resultó positiva para carcinoma urotelial.

\section{DISCUSIÓN}

La disnea en los pacientes con neoplasia puede tener múltiples causas que incluyen infecciones, TEP, metástasis o to- 

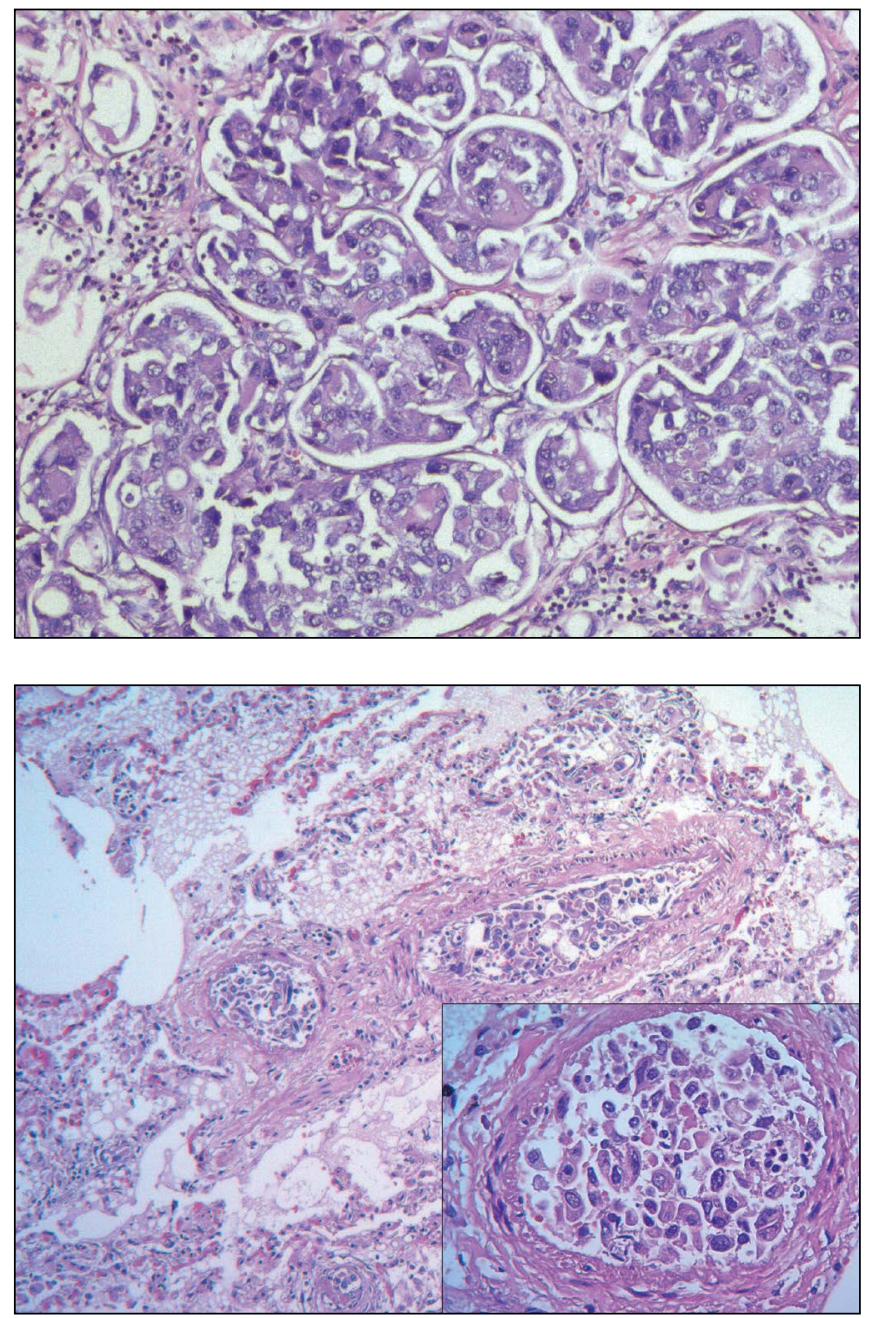

Figura 1. Células neoplásicas con elevada atipia nuclear formando pequeños nidos y micropapilas rodeadas por un estroma típicamente retraído.

Figura 2. a) Émbolos tumorales en la microvasculatura del parénquima pulmonar. b) Imagen a gran aumento en la que se observan las células uroteliales neoplásicas en el interior de una arteriola pulmonar. xicidad por diferentes tratamientos como quimioterapia o radioterapia. Los embolismos tumorales pulmonares (ETP) son una causa poco frecuente y su diagnóstico todavía resulta más difícil cuando la neoplasia no se conoce previamente ${ }^{3}$.

En series autópsicas se han descrito ETP hasta en el 3-26\% de pacientes con tumores sólidos, pero solo en un $8 \%$ su hallazgo influyó de forma significativa en la mortalidad $^{2,4}$. En una amplia serie japonesa de revisión de 65.181 autopsias en pacientes con cáncer, se encontró cualquier tipo de embolismo pulmonar en un $2,62 \%$ de casos, correspondiendo el $88,6 \%$ a émbolos pulmonares trombóticos. Sólo se evidenció ETP en el $0,19 \%$ de las autopsias ${ }^{5}$. Los tumores más frecuentemente implicados eran mama, pulmón y estómago ${ }^{1}$, aunque en otras series de países orientales el hepatoma $^{6}$, el ovario e incluso el riñón resultaron los más frecuentes ${ }^{5}$. Se han descrito casos aislados en tumores de vejiga ${ }^{5,7}$, pero no hemos encontrado ningún caso publicado en tumores uroteliales de vías superiores como nuestro paciente. Algunos autores consideran que la histología del tumor influye en su aparición y así en la serie de Sakuma ${ }^{5}$ la mayor incidencia de ETP se encontró en los casos de carcinoma de 
células grandes, adenocarcinoma y carcinoma hepatocelular y cuando el tumor estaba presente en el aparato digestivo y en el hígado. En nuestro caso, pensamos que, efectivamente, el subtipo histológico poco frecuente dentro de los carcinomas transicionales $^{8}$, influyó en la aparición de ETP, ya que se trata de una variante muy agresiva, con marcado tropismo vascular.

Se considera que los ETP provienen de tumores sólidos que invaden la circulación sistémica, son filtrados y se depositan en la microvasculatura pulmonar ${ }^{9}$. Las células tumorales pueden afectar a la vasculatura pulmonar de varias formas: émbolos proximales grandes ${ }^{10}$, diseminación linfangítica, enfermedad microvascular pura (como en nuestro caso) o combinación de varias ${ }^{1}$.

Su presentación clínica más frecuente es la disnea ${ }^{1}$; a menudo se confunde con el TEP y característicamente las pruebas realizadas pueden ser normales (Rx torax, TC, angiografía pulmonar). En la TC con contraste se pueden apreciar obstrucciones y dilataciones de ramas subsegmentarias, líneas subpleurales y opacidades en forma de cuña por infarto pulmonar, además de signos de hipertensión venosa pulmonar y otros hallazgos que confirmen la presencia de neoplasia ${ }^{11}$. En nuestro paciente no pudo realizarse por alergia a contrastes yodados. La GV/Q puede ser normal y en ocasiones muestra pequeños defectos de perfusión simétricos y periféricos, a diferencia del TEP ${ }^{11}$. La elevación del Dímero-D no resulta de ayuda ya que puede elevarse por la propia neoplasia o por activación de la cascada de la coagulación que acompaña a los propios trombos pulmonares2.

Debe sospecharse el diagnóstico de ETP en pacientes con cáncer y disnea subaguda en los que se sospecha TEP y no se puede confirmar su origen trombótico. El cateterismo derecho con citología obtenida en arteria pulmonar ${ }^{1,9}$ o la biopsia pulmonar ${ }^{2}$ podrían permitir su diagnóstico, pero no siempre es fácil su realización por problemas técnicos o por la mala situación clínica del paciente. No obstante, el pronóstico de los pacientes con ETP es muy malo, con una elevadísima mortalidad, incluso en los casos con confirmación diagnóstica en vida y en la mayor parte de los pacientes se establece el diagnóstico mediante estudio necrópsico.

\section{BIBLIOGRAFÍA}

1. Roberts KE, Hamele-Bena D, SaQi A, Stein CA, Cole RP. Pulmonary tumor embolism/ A review of the literature. Am J Med 2003; 115: 228-232.

2. Kridel R, Myit S, Pache JC, Gaspoz JM. Pulmonary tumor embolism/ a rare cause of acute right heart failure with elevated D-dimers. J Thorac Oncol 2008; 3: 1482-1483.

3. Gutiérrez Macías A, Barandiarán KE, Ercoreca FJ, DE ZÁRATE MM. Acute cor pulmonale due to microscopic tumour embolism as the first manifestation of hepatocellular carcinoma. Eur J Gastroenterol Hepatol 2002; 14: 775777.

4. Winterbauer RH, ElfEnbein IB, Ball WC JR. Incidence and clinical significance of tumor embolization to the lungs. Am J Med 1968; 45: 271-290.

5. Sakuma M, FukUI S, NaKamura M, Takahashi T, KITAMUKAI O, YAZU T et al. Cancer and pulmonary embolism. Thrombotic embolism, rumor embolism, and tumor invasión into a large vein. Circ J 2006; 70: 744-749.

6. Chan CK, Hutcheon MA, Hyland RH, Smith GJ, Patterson BJ, Matthay RA. Pulmonary tumor embolism/a critical review of clinical, imaging, and hemodynamic features. J Thorac Imag 1987; 2: 4-14.

7. Chertcoff FJ, Emery NC, Villagomez R, Veltri MA, Venditti Y, Young P et al. Embolismo pulmonar tumoral/reporte de dos casos. Rev Med Chile 2009; 137: 1613-1616.

8. Sung HH, Cho J, Kwon GY, Jeon HG, Jeong BC, SEO SI et al. Clinical significance of micropapillary urothelial carcinoma of the upper urinary tract. J Clin Pathol 2014; 67: 49-54.

9. Babar SI, Sobonya RE, SNyder LS. Pulmonary microvascular cytology for the diagnosis of pulmonary tumor embolism. West J Med 1998; 168: 47-50.

10. Shimada S, Saito H, Arai Y. Pulmonary embolism caused by spontaneous migration of tumor thrombus of renal cell carcinoma/a report of two cases. Int J Urol 2012; 19: 277-278.

11. Varona D, Andreu J, Pallisa E, Persiva O, Roque A. Patología vascular torácica en pacientes oncológicos. Radiología 2011; 53: 335-348. 